\title{
Searches for diffuse X-ray emission around millisecond pulsars: an X-ray nebula associated with PSR J2124-3358
}

\author{
C. Y. Hui and W. Becker
}

Max-Planck Institut für Extraterrestrische Physik, Giessenbachstrasse 1, 85741 Garching bei München, Germany

Received 21 November 2005 / Accepted 19 January 2006

\section{ABSTRACT}

We report on diffuse X-ray emission associated with the nearby solitary millisecond pulsar PSR J2124-3358 detected with XMM-Newton and Chandra. The emission extends from the pulsar to the northwest by $\sim 0.5$ arcmin. The spectrum of the nebular emission can be modeled with a power-law of photon index $2.2 \pm 0.4$, in line with the emission originating from accelerated particles in the post shock flow. For PSR J0437-4715, PSR J0030+0451 and PSR J1024-0719, which all have spin parameters comparable to that of PSR J2124-3358, no diffuse emission is detected down to a $3-\sigma$ limiting flux of $\sim 4-7 \times 10^{-15} \mathrm{erg} \mathrm{s}^{-1} \mathrm{~cm}^{-2}$.

Key words. pulsars: individual: PSR J2124-3358, J0437-4715, J0030+0451, J1024-0719 - stars: neutron - X-rays: stars

\section{Introduction}

Diffuse plerionic emission is often a special marker of young and powerful pulsars if their spin-down energy is in excess of $\sim 10^{36} \mathrm{erg} / \mathrm{s}$. Extended diffuse emission associated with older and less powerful pulsars, however, is rare and so far only seen in Geminga (Caraveo et al. 2003), PSR B1929+10 (Becker et al. 2005), PSR B2224+65 (Romani et al. 1997; Chatterjee \& Cordes 2002) and the millisecond pulsars (MSPs) PSR B1957+20 (Stappers et al. 2003), J0437-4715 (Bell et al. 1995), and J2124-3358 (Gaensler et al. 2002). While all three MSPs have bright bow-shock nebulae detected in $\mathrm{H}_{\alpha}$, diffuse X-ray emission associated with it could be detected only from the black widow pulsar PSR B1957+20 in a recent Chandra observation (Stappers et al. 2003). Comparable deep observations of almost all X-ray bright MSPs, however, have been performed by XMM-Newton in previous years. These XMM-Newton data thus provides us a valuable data base to search for diffuse and extended emission components from these MSPs as well.

In this letter we report on a search for diffuse X-ray emission to be associated with the MSPs PSR J2124-3358, J0437-4715, J0030+0451 and J1024-0719. All these pulsars have comparable spin parameters (cf. Table 1) so that differences in their emission properties are most likely cause by differences in the pulsar-ISM interaction/local environment rather than by differences in their total energy output.

PSR J2124-3358 and J1024-0719 were both discovered during the Parkes $436 \mathrm{MHz}$ survey of the southern sky (Bailes et al. 1997). PSR J0030+0451 was discovered at $430 \mathrm{MHz}$ during the Arecibo Drift Scan Search (Somer 2000) and independently in the Bologna sub-millisecond pulsar survey
(D'Amico 2000), whereas PSR J0437-4715 was discovered in the Parkes southern sky survey (Johnston et al. 1993). PSR J0437-4715 was the first MSP of which pulsed X-ray emission was detected (Becker \& Trümper 1993). X-ray emission from PSR J2124-3358 and J1024-0719 was reported by Becker \& Trümper $(1998,1999)$ in ROSAT HRI data whereas the X-ray counterpart of PSR J0030+0451 was discovered in the final ROSAT PSPC observation (Becker et al. 2000). $\mathrm{A}_{\alpha}$ bow shock is seen around PSR J0437-4715 (Bell et al. 1995). However, its X-ray counterpart was not detected by ROSAT and Chandra (Becker \& Trümper 1999; Zavlin et al. 2002). Gaensler et al. (2002) discovered an $\mathrm{H}_{\alpha}$-emitting bow shock nebula around PSR J2124-3358. This bow shock is very broad and highly asymmetric about the direction of the pulsar's proper motion. The asymmetric shape might be caused by a significant density gradient in the ISM, bulk flow of ambient gas and/or anisotropies in the pulsar's relativistic wind (Gaensler et al. 2002). Observation of PSR J0030+0451, performed with the ESO NTT in La Sila, did not detect diffuse $\mathrm{H}_{\alpha}$ emission associated with it (A. Pellizzoni priv. com.).

\section{Observations and data analysis}

The pulsars PSR J2124-3358, J0030+0451, J1024-0719 and J0437-4715 were observed by XMM-Newton in 2002 April 14-15, in 2001 June 19-20, in 2003 December 2 and in 2002 October 9, respectively. In all observations the MOS1/2 cameras were operated in full-frame mode while the PN camera was setup to work in the fast-timing mode. The PN fasttiming mode provides only limited spatial information so that these data are not used in the present analysis. To block stray 
Table 1. Pulsar parameters of PSRs J0030+0451, J2124-3358, J1024-0719 and J0437-4715 (from Manchester et al. 2005).

\begin{tabular}{lcccc}
\hline \hline Pulsar & PSR J0030+0451 & PSR J2124-3358 & PSR J1024-0719 & PSR J0437-4715 \\
\hline Right Ascension $(\mathrm{J} 2000)$ & $00^{\mathrm{h}} 30^{\mathrm{m}} 27.432^{\mathrm{s}}$ & $21^{\mathrm{h}} 24^{\mathrm{m}} 43.862^{\mathrm{s}}$ & $10^{\mathrm{h}} 24^{\mathrm{m}} 38.700^{\mathrm{s}}$ & $04^{\mathrm{h}} 37^{\mathrm{m}} 15.787^{\mathrm{s}}$ \\
Declination $(\mathrm{J} 2000)$ & $+04^{\circ} 51^{\prime} 39.7^{\prime \prime}$ & $-33^{\circ} 58^{\prime} 44.257^{\prime \prime}$ & $-07^{\circ} 19^{\prime} 18.915^{\prime \prime}$ & $-47^{\circ} 15^{\prime} 08.462^{\prime \prime}$ \\
Pulsar Period, $P(\mathrm{~ms})$ & 4.865453207369 & 4.9311148591481 & 5.1622045539088 & 5.7574518310720 \\
Period derivative $\dot{P}\left(10^{-20} \mathrm{~s} \mathrm{~s}^{-1}\right)$ & 1.0 & 1.33 & 1.85 & 1.87 \\
Age $\left(10^{9}\right.$ yr) & 7.71 & 5.86 & 4.41 & 4.89 \\
Surface dipole magnetic field $\left(10^{8} \mathrm{G}\right)$ & 2.23 & 2.60 & 3.13 & 3.32 \\
Epoch of Period (MJD) & 50984.4 & 50288.0 & 51018.0 & 51194.0 \\
Dispersion Measure $\left(\mathrm{pc} \mathrm{cm} \mathrm{cm}^{-3}\right)$ & 4.3328 & 4.6152 & 6.491 & 2.6469 \\
Dispersion based distance $(\mathrm{pc})$ & 230 & 250 & 350 & 140 \\
Spin-down Luminosity $\left(10^{33}\right) \mathrm{erg} \mathrm{s}^{-2}$ & 3.43 & 4.38 & 5.3 & 3.87 \\
\hline
\end{tabular}

light and optical leakage from bright foreground stars we operated the MOS1/2 cameras with the thin filters during the observations of PSRs J0030+0451, J1024-0719 and J0437-4715 while PSR J2124-3358 was observed by using a medium filter. Events were selected for the energy range $0.25-10 \mathrm{keV}$ and standard correction procedures were applied to reduce the data (e.g. Becker et al. 2005). Filtering the data for times of excessive background from soft proton flares was done using conservative thresholds in view of the planned search for diffuse and extended X-ray emission. The effective exposure time after data reduction turns out to be $40 \mathrm{ks}$ for PSR J2124-3358, $14 \mathrm{ks}$ for J0030+0451, $65 \mathrm{ks}$ for J1024-0719 and $55 \mathrm{ks}$ for J0437-4715.

A vignetting corrected image of the field of PSR J21243358 as seen by the XMM-Newton's MOS1/2 CCDs is shown in Fig. 1a. The binning factor in this image is 6 arcsec. Adaptive smoothing with a Gaussian kernel of $\sigma<4$ pixels has been applied to the image. X-ray contours are calculated and overlaid on the image. The contour lines are at the levels of $(4.2,5.2,7.6,13,28,63) \times 10^{-6}$ cts s $^{-1} \operatorname{arcsec}^{-2}$. It can be seen that the X-ray source which is coincident with the pulsar position has an asymmetric source structure of $\sim 0.5 \mathrm{arcmin}$ extent, with its orientation to the northwest. Systematic effects which could cause an adequate distortion of the instrument's point spread function (PSF) are not known for XMM-Newton. We are therefore prompted to interpret this elongated structure in terms of a pulsar X-ray trail. The net count rate of the diffuse emission in a $30 \times 35$ arcsec box centered at $\mathrm{RA}(\mathrm{J} 2000)=21^{\mathrm{h}} 24^{\mathrm{m}} 42.69^{\mathrm{s}}$, Dec $=-33^{\circ} 58^{\prime} 16.06^{\prime \prime}$ and oriented along the extended feature is estimated to be $(1.25 \pm 0.16) \times 10^{-3}$ cts s $^{-1}$. For this estimate we determined the background from a low count region close to PSR J2124-3358. For comparison, the pulsar emission is estimated to have a net count rate of $(1.07 \pm 0.01) \times 10^{-2} \mathrm{cts} \mathrm{s}^{-1}$ in a circle of 18 arcsec radius centered at the pulsar position. The signal-to-noise of this elongated feature is $\sim 4$ in the energy range $0.25-5 \mathrm{keV}$.

PSR J2124-3358 was observed by Chandra with the backilluminated ACIS-S3 chip in the focus of the XRT. The observation took place on 2004 December 19-20 for an exposure time of $\sim 30 \mathrm{ks}$. An image made from this data with 0.5 arcsec binning and adaptive smoothing applied (using a Gaussian kernel with $\sigma<1.5$ pixel) is shown in Fig. 1b. Arc-like diffuse emission which is within the pulsar's $\mathrm{H}_{\alpha}$ nebula is clearly detected. Its net count rate is estimated to be
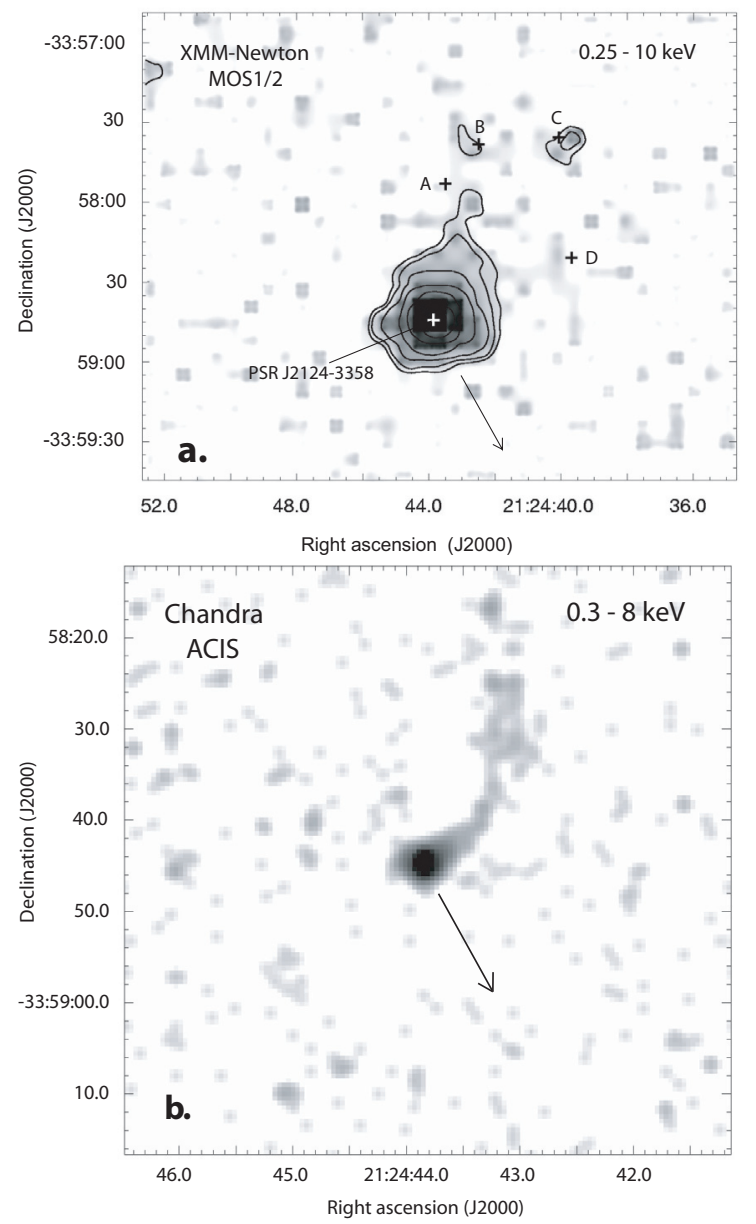

Fig. 1. a) XMM-Newton MOS1/2 image of PSR J2124-3358 with overlaid contours. The pulsar proper motion is indicated by an arrow. The position of bright stars located in the 1.5 arcmin neighborhood of PSR J2124-3358 are indicated. b) PSR J2124-3358 as seen by the ACIS detector aboard Chandra.

$(1.28 \pm 0.23) \times 10^{-3}$ cts s$^{-1}$ in a box of $10 \times 20$ arcsec centered at $\mathrm{RA}(\mathrm{J} 2000)=21^{\mathrm{h}} 24^{\mathrm{m}} 43.17^{\mathrm{s}}$, Dec $=-33^{\circ} 58^{\prime} 33.53^{\prime \prime}$. With the aid of PIMMS, the pileup fraction of the ACIS-S3 data was found to be less than $0.2 \%$, i.e. is negligible. The signal-tonoise of this feature is $\sim 5$ in the energy range of $0.3-8 \mathrm{keV}$.

Since the PSF width of XMM-Newton is about 10 times that of Chandra, it blurred most of the detailed structure seen in the Chandra data. However, it should be noted that the overall 
Table 2. Identifications of the stars around the X-ray trail of PSR J2124-3358.

\begin{tabular}{lccc}
\hline \hline Stars $^{a}$ & USNO catalogue ID & Magnitude: $R$ & Magnitude: $B$ \\
\hline A & U052543254607 & 17.1 & 18.7 \\
B & U052543254423 & 18.0 & 20.2 \\
C & U052543253997 & 16.7 & 17.4 \\
D & U052543253910 & 13.5 & 15.5 \\
\hline
\end{tabular}

${ }^{a}$ See Fig. 1a.

direction of the feature in the Chandra image is consistent with the orientation of the trail detected by XMM-Newton.

In order to investigate a possible contribution to the diffuse X-ray emission by nearby stars we investigated the Digitized Sky Survey data (DSS) for the sky region around PSR J2124-3358. There are four field stars (labeled as A, B, C and D in Fig. 1a and Table 2) in the 1.5 arcmin neighborhood of PSR J2124-3358. From the USNO catalogue, we identified the magnitudes of these stars. None of them is found to match the position of the diffuse elongated X-ray structure seen in the XMM-Newton and Chandra data. It can be seen in Fig. 1a that the positions of stars $\mathrm{B}$ and $\mathrm{C}$ coincide with two faint $\mathrm{X}$-ray sources which are disconnected with the trail emission of PSR J2124-3358 though.

To examine the nature of the faint X-ray nebular emission by a spectral analysis we make use of the XMM-Newton data and extract the energy spectrum from within a $30 \times 35$ arcsec box. Using a box rather than a circular selection region allows to avoid the emission from the pulsar and excludes any potential contamination from the field stars B and C. However, we estimate that the wing of the XMM-Newton PSF centered at the pulsar position still contributes $\sim 20 \%$ to the total counts inside the box. The background spectrum was extracted from a source free region near to PSR J2124-3358. In total, 92 and 67 source counts are available from the trail in the MOS1 and MOS2 detectors, respectively. Response files were computed by using the XMMSAS tasks RMFGEN and ARFGEN. The spectra were dynamically binned so as to have at least 10 counts per bin.

In the Chandra data we selected the energy spectrum of the diffuse emission from a box of size $10 \times 20$ arcsec. Owing to the narrow PSF of Chandra the contamination of pulsar emission in this box is negligible. The background spectrum was extracted from a low count region near to the diffuse feature. In total 46 source counts are contributed from the Chandra data. Response files were computed by using tools MKRMF and MKARF of CIAO. The spectrum was binned to have at least 8 counts per bin. The degradation of quantum efficiency of ACIS was corrected by applying the XSPEC model ACISABS.

We hypothesize that the diffused emission should be originated from the interaction of pulsar wind and the ISM. Synchrotron radiation from the ultra-relativistic electrons is generally believed to be the emission mechanism of the pulsar wind nebula, which then is characterized by a powerlaw spectrum. With a view to test this hypothesis, we fitted an absorbed power-law model to the nebular spectrum with XSPEC 11.3 .1 in the $0.25-10 \mathrm{keV}$ energy range. With a

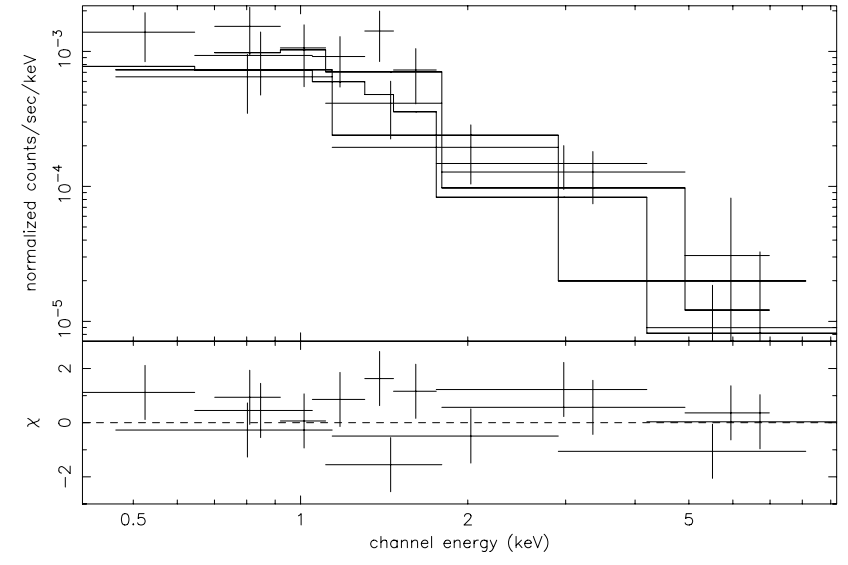

Fig. 2. Energy spectrum of the X-ray trail of PSR J2124-3358 as observed with MOS1/2 and ACIS-S3 detectors and simultaneously fitted to an absorbed power-law model (upper panel) and contribution to the $\chi^{2}$ fit statistic (lower panel).

column density of $5 \times 10^{20} \mathrm{~cm}^{-2}$ as obtained from spectral fits to the pulsar emission, we found that the model describes the observed spectrum fairly well $\left(\chi_{v}^{2}=0.79\right.$ for 26 d.o.f.). The photon index is $\alpha=2.2 \pm 0.4$ and the normalization at $1 \mathrm{keV}$ is $(2.94 \pm 0.48) \times 10^{-6}$ photons $\mathrm{keV}^{-1} \mathrm{~cm}^{-2} \mathrm{~s}^{-1}(1-\sigma$ error for 1 parameter in interest). In view of the low photon statistic we tested for a possible dependence of the fitted model parameters against the background spectrum. All deviations found in repeating the fits with different background spectra were within the $1-\sigma$ confidence interval quoted above. The unabsorbed fluxes and luminosities deduced for the best fit model parameters and the energy ranges $0.1-2.4 \mathrm{keV}$ and $0.5-10 \mathrm{keV}$ are $f_{\mathrm{X}}=1.8 \times 10^{-14} \mathrm{erg} \mathrm{s}^{-1} \mathrm{~cm}^{-2}, L_{\mathrm{X}}=1.3 \times 10^{29} \mathrm{erg} \mathrm{s}^{-1}$ and $f_{\mathrm{X}}=1.2 \times 10^{-14} \mathrm{erg} \mathrm{s}^{-1} \mathrm{~cm}^{-2}, L_{\mathrm{X}}=8.9 \times 10^{28} \mathrm{erg} \mathrm{s}^{-1}$, respectively. The best fitting spectral model is displayed in Fig. 2.

Motivated by the extended source structure seen in PSR J2124-3358 we have also inspected the XMM-Newton data of PSRs J0030+0451, J1024-0719 and J0437-4715 in order to search for extended tail-like X-ray emission. There is no evidence for any extended emission around these pulsars. In order to deduce an upper limit for an X-ray trail in these sources we have estimated the net count rates for the regions of $30 \times 35$ arcsec right behind the corresponding directions of each pulsar's proper motion. The net count rate for any diffuse emission in these regions is $(3.1 \pm 1.5) \times 10^{-4} \mathrm{cts} \mathrm{s}^{-1}$, $(3.1 \pm 0.7) \times 10^{-4} \mathrm{cts} \mathrm{s}^{-1}$ and $(2.5 \pm 0.9) \times 10^{-4} \mathrm{cts} \mathrm{s}^{-1}$ for PSRs J0030+0451, J1024-0719 and J0437-4715, respectively. The quoted errors are $1-\sigma$ confidence intervals. Assuming a Crablike spectrum (i.e. $\alpha=2$ ) and taking the values of column density inferred from the pulsar spectral fits $\left(1 \times 10^{20} \mathrm{~cm}^{-2}\right.$ for $\mathrm{J} 0030+0451,2 \times 10^{20} \mathrm{~cm}^{-2}$ for J1024-0719 and $3 \times 10^{19} \mathrm{~cm}^{-2}$ for J0437-4715), we calculated the $3-\sigma$ limiting fluxes from the above count rates. For PSRs J0030+0451, J1024-0719 and J0437-4715, the limiting fluxes in $0.5-10 \mathrm{keV}$ are estimated to be $6.9 \times 10^{-15} \mathrm{erg} \mathrm{s}^{-1} \mathrm{~cm}^{-2}, 4.9 \times 10^{-15} \mathrm{erg} \mathrm{s}^{-1} \mathrm{~cm}^{-2}$ and $4.2 \times 10^{-15} \mathrm{erg} \mathrm{s}^{-1} \mathrm{~cm}^{-2}$, respectively. 
Table 3. Properties of rotation-powered pulsars with $\mathrm{X}$-ray $/ \mathrm{H}_{\alpha}$ bow shocks.

\begin{tabular}{|c|c|c|c|c|c|c|c|c|}
\hline PSR & $\begin{array}{c}\text { Other name/ } \\
\text { associated SNR }\end{array}$ & $\begin{array}{c}\dot{E} \\
\mathrm{erg} \mathrm{s}^{-1}\end{array}$ & $\begin{array}{c}L_{\mathrm{X}}^{\mathrm{diff}}(0.5-10 \mathrm{keV})^{a} \\
\mathrm{erg} \mathrm{s}^{-1}\end{array}$ & $\begin{array}{c}\tau_{\text {spin-down }} \\
\text { years }\end{array}$ & $\begin{array}{c}\mathrm{H}_{\alpha} \\
\text { bow shock }\end{array}$ & $\begin{array}{c}\text { X-ray } \\
\text { trail }\end{array}$ & $\begin{array}{l}\text { Radio } \\
\text { nebula }\end{array}$ & Reference \\
\hline \multicolumn{9}{|c|}{ Young pulsars } \\
\hline J0537-6910 & LMC: N157B & $4.8 \times 10^{38}$ & $3.5 \times 10^{36}$ & $5.0 \times 10^{3}$ & - & $\mathrm{d}$ & - & 1,2 \\
\hline B1757-24 & Duck & $2.6 \times 10^{36}$ & $4.6 \times 10^{32}$ & $1.6 \times 10^{4}$ & - & $\mathrm{d}$ & $\mathrm{d}$ & 3 \\
\hline B1853+01 & W44 & $4.3 \times 10^{35}$ & $5.7 \times 10^{32}$ & $2.0 \times 10^{4}$ & - & $\mathrm{d}$ & $\mathrm{d}$ & 4 \\
\hline $\mathrm{J} 1747-2958$ & Mouse & $2.5 \times 10^{36}$ & $5.3 \times 10^{34}$ & $2.6 \times 10^{4}$ & - & $\mathrm{d}$ & $\mathrm{d}$ & 5 \\
\hline B1951+32 & CTB80 & $3.7 \times 10^{36}$ & $2.5 \times 10^{33}$ & $1.1 \times 10^{5}$ & $\mathrm{~d}$ & $\mathrm{~d}$ & $\mathrm{~d}$ & $6,7,8$ \\
\hline \multicolumn{9}{|c|}{ Middle age and old pulsars } \\
\hline B0740-28 & & $1.4 \times 10^{35}$ & - & $1.6 \times 10^{5}$ & $\mathrm{~d}$ & - & - & 9 \\
\hline B0633+17 & Geminga & $3.2 \times 10^{34}$ & $8.9 \times 10^{28}$ & $3.4 \times 10^{5}$ & - & $\mathrm{d}$ & - & 10 \\
\hline B2224+65 & Guitar & $1.2 \times 10^{33}$ & $3.7 \times 10^{31}$ & $1.1 \times 10^{6}$ & $\mathrm{~d}$ & $?$ & - & 11,12 \\
\hline $\mathrm{B} 1929+10$ & & $3.9 \times 10^{33}$ & $8.3 \times 10^{29}$ & $3.1 \times 10^{6}$ & - & $\mathrm{d}$ & $?$ & 13 \\
\hline \multicolumn{9}{|c|}{ Millisecond pulsars } \\
\hline B1957+20 & & $1.1 \times 10^{35}$ & $1.9 \times 10^{31}$ & $2.2 \times 10^{9}$ & $\mathrm{~d}$ & $\mathrm{~d}$ & - & 14 \\
\hline J0437-4715 & & $3.9 \times 10^{33}$ & - & $4.9 \times 10^{9}$ & $\mathrm{~d}$ & - & - & 15,16 \\
\hline $\mathrm{J} 2124-3358$ & & $4.4 \times 10^{33}$ & $10^{29}$ & $5.9 \times 10^{9}$ & $\mathrm{~d}$ & $\mathrm{~d}$ & - & 17,18 \\
\hline
\end{tabular}

${ }^{a}$ The $\mathrm{X}$-ray luminosities of the diffuse emission are taken from the corresponding reference and recalculate into energy band of $0.5-10 \mathrm{keV}$ for easy comparison. References: (1) Wang \& Gotthelf (1998); (2) Wang et al. (2001); (3) Kaspi et al. (2001); (4) Petre et al. (2002); (5) Gaensler et al. (2004); (6) Migliazzo et al. (2002); (7) Moon et al. (2004); (8) Li et al. (2005); (9) Jones et al. (2002); (10) Caraveo et al. (2003); (11) Romani et al. (1997); (12) Chatterjee \& Cordes (2002); (13) Becker et al. (2005); (14) Stappers et al. (2003); (15) Bell et al. (1995); (16) Zavlin et al. (2002); (17) Gaensler et al. (2002); (18) this letter; and Manchester et al. (2005) otherwise.

\section{Discussion and conclusion}

Basic characteristics of the X-ray bow shocks detected around rotation-powered pulsars are summarized in Table 3. PSR J2124-3358 is a new candidate to this group. Its tail is found to have an extent of $\sim 0.5$ arcmin. Adopting the distance of $\sim 250 \mathrm{pc}$, the tail has a length of $l \sim 1.1 \times 10^{17} \mathrm{~cm}$. For the pulsar's proper motion velocity of $58 \mathrm{~km} \mathrm{~s}^{-1}$ (Manchester et al. 2005), the timescale, $t_{\text {flow }}$, for the passage of the pulsar over the length of its X-ray trail is estimated to be $\sim 600 \mathrm{yrs}$. According to the discussion in Becker et al. (2005) on the trail emission of PSR B1929+10 we estimate the magnetic field in the shocked region by assuming $t_{\text {flow }}$ to be comparable to the electron lifetime of the synchrotron emission. This yields $\sim 30 \mu \mathrm{G}$ for the inferred magnetic field strength in the emitting region. The magnetic field strength in the ISM is estimated to be $\sim 2-6 \mu \mathrm{G}$ (cf. Beck et al. 2003, and references therein). Taking into account that the magnetic field in the termination shock might be compressed (e.g. Kennel \& Coroniti 1984), our estimation is approximately consistent if the compression factor is $\sim 7$.

Following Becker et al. (2005), we apply a simple one zone model (Chevalier 2000) to estimate the spectral behavior and the X-ray luminosity of the nebular emission. The X-ray luminosity and spectral index depend on the inequality between the characteristic observed frequency $v_{X}^{\text {obs }}$ and the electron synchrotron cooling frequency $v_{\mathrm{c}}$ which is estimated to be $1.6 \times 10^{17} \mathrm{~Hz}$. Since in general $v_{\mathrm{X}}^{\text {obs }}>v_{\mathrm{c}}$, we concluded that the emission is in a fast cooling regime. Electrons with the energy distribution, $N(\gamma) \propto \gamma^{-p}$, are able to radiate their energy in the trail with photon index $\alpha=(p+2) / 2$. The index $p$ due to shock acceleration typically lies between 2 and 3 (cf. Cheng et al. 2004, and references therein). Taking $p=2.35$ yields $\alpha^{\text {th }} \simeq 2.2$ which is in accordance with the result from the observed value $\alpha^{\text {obs }}=2.2 \pm 0.4$. Assuming the energy equipartition between the electron and proton (Cheng et al. 2004), we take the fractional energy density of electron $\epsilon_{\mathrm{e}}$ to be $\sim 0.5$ and the fractional energy density of the magnetic field $\epsilon_{B}$ to be $\sim 0.01$. Assuming a number density of ISM to be $1 \mathrm{~cm}^{-3}$, the distance of the shock from the pulsar is estimated to be $\sim 3.6 \times 10^{16} \mathrm{~cm}$. With these estimates, the calculated luminosity, $v L_{v}$, is given as $\sim 10^{29} \mathrm{erg} \mathrm{s}^{-1}$ which is well consistent with the observed values of $1.3 \times 10^{29} \mathrm{erg} \mathrm{s}^{-1}(0.1-2.4 \mathrm{keV})$ and $8.9 \times 10^{28} \mathrm{erg} \mathrm{s}^{-1}(0.5-10 \mathrm{keV})$.

Although the general properties of the X-ray trail in PSR J2124-3358 are not in contradiction with properties observed in other pulsars there are still ambiguities which are not completely resolved. First, one should notice that the trail is misaligned with the direction of the pulsar's proper motion. As reported by Gaensler et al. (2002), the head of the $\mathrm{H}_{\alpha}$ bow shock is found to be highly asymmetric about the pulsar's velocity vector with the apparent nebular symmetry axis deviated from the velocity vector by $\sim 30^{\circ}$. Even though the misalignment of the X-ray trail seems to agree with the asymmetry of the $\mathrm{H}_{\alpha}$ nebula, deeper observations by XMM-Newton and Chandra are required in order to constrain the physical properties of this interesting nebula in higher detail. 
Acknowledgements. We thank the referee Giancarlo Cusumano for thoroughly reading the manuscript and the many useful comments. We acknowledge the use of data obtained with XMM-Newton and Chandra. XMM-Newton is an ESA science mission with instruments and contributions directly funded by ESA Member States and NASA.

\section{References}

Bailes, M., Johnston, S., Bell, J. F., et al. 1997, ApJ, 481, 386

Beck, R., Shukurov, A., Sokoloff, D., \& Wielebinski, R. 2003, A\&A, 411, 99

Becker, W., \& Trümper, J. 1993, Nature, 365, 528

Becker, W., \& Trümper, J. 1998, IAU Circ., 6829

Becker, W., \& Trümper, J. 1999, A\&A, 341, 803

Becker, W., Trümper, J., Lommen, A. N., \& Backer, D. C. 2000, ApJ, 545,1015

Becker, W., Kramer, M., Jessner, A., et al. 2005, ApJ, in press [arXiv: astro-ph/0506545]

Bell, J. F., Bailes, M., Manchester, R. N., Weisberg, J. M., \& Lyne, A. G. 1995, ApJ, 440, L81

Caraveo, P. A., Bignami, G. F., DeLuca, A., et al. 2003, Science, 301, 1345

Chatterjee, S., \& Cordes, J. M. 2002, ApJ, 575, 407

Cheng, K. S., Taam, R. E., \& Wang, W. 2004, ApJ, 617, 480

Chevalier, R. A. 2000, ApJ, 539, L45

D’Amico 2000, N., in Pulsar Astronomy - 2000 and Beyond, 202th ASP Conf. Ser., ed. M. Kramer, N. Wex, \& R. Wielebinski (San Francisco: ASP), 27
Gaensler, B. M., van der Swaluw, E., Camilo, F., et al. 2004, ApJ, 616, 383

Gaensler, B. M., Jones, D. H., \& Stappers, B. W. 2002, ApJ, 580, L137 Johnston, S., Lorimer, D. R., Harrison, P. A., et al. 1993, Nature, 361, 613

Jones, D. H., Stappers, B. W., \& Gaensler, B. M. 2002, A\&A, 389, L1 Kaspi, V. M., Gotthelf, E. V., Gaensler, B. M., \& Lyutikov, M. 2001, ApJ, 562, L163

Kennel, C. F., \& Coroniti, F. V. 1984, ApJ, 283, 694

Li, X. H., Lu, F. J., \& Li, T. P. 2005, ApJ, 628, 931

Manchester, R. N., Hobbs, G. B., Teoh, A., \& Hobbs, M. 2005, AJ, 129,1993

Migliazzo, J. M., Gaensler, B. M., Backer, D. C., et al. 2002, ApJ, 567, L141

Moon, D.-S., Lee, J.-J., Eikenberry, S. S., et al. 2004, ApJ, 610, L33

Petre, R., Kuntz, K. D., \& Shelton, R. L. 2002, ApJ, 579, 404

Romani, R. W., Cordes, J. M., \& Yadigaroglu, I.-A. 1997, ApJ, 484, L137

Somer, A. 2000, in Pulsar Astronomy - 2000 and Beyond, 202th ASP Conf. Ser., ed. M. Kramer, N. Wex, \& R. Wielebinski (San Francisco: ASP), 17

Stappers, B. W., Gaensler, B. M., Kaspi, V. M., van der Klis, M., \& Lewin, W. H. G. 2003, Science, 299, 1372

Wang, Q. D., \& Gotthelf, E. V. 1998, ApJ, 494, 623

Wang, Q. D., Gotthelf, E. V., Chu, Y.-H., \& Dickel, J. R. 2001, ApJ, 559,275

Zavlin, V. E., Pavlov, G. G., Sanwal, D., et al. 2002, ApJ, 569, 894 